\title{
ON THE SERIAL COMPLETION OF DELETED SCHAUDER BASES BY DOMAIN ADJUSTMENT
}

\author{
BEN-AMI BRAUN
}

\begin{abstract}
Given a Schauder basis in a Banach function space of a specified type, one can delete any finite number of elements of the basis and still preserve serial totality by making an arbitrarily small adjustment of the domain.
\end{abstract}

Let $\left\{\phi_{n}\right\}_{n=1}^{\infty}$ be a system of real valued functions finite almost everywhere and measurable on a set $G \subset[0,1]$, mes. $G>0$. Talalyan [2], [3] proved the following to be equivalent:

(a) $\left\{\phi_{n}\right\}_{n=1}^{\infty}$ is total in measure on $G$, that is, for every measurable function $f$ defined on $G$, there exists a sequence of finite linear combinations of functions of the system $\left\{\phi_{n}\right\}_{n=1}^{\infty}$ which converges in measure to $f$ on $G$.

(b) For each positive number $\varepsilon$, there is a measurable set $S_{\varepsilon}$ whose measure exceeds $1-\varepsilon$, such that $\left\{\phi_{n}\right\}_{n=1}^{\infty}$ is total in $L^{2}\left(S_{\varepsilon}\right)$.

We shall say that $\left\{\phi_{k}\right\}_{k=1}^{\infty}$ is serially total in some function space, if for any given function $f$ in the space we can find a series $\sum_{k=1}^{\infty} a_{k} \phi_{k}$ which converges to $f$ in the metric of the space. The result of this paper can be viewed as a first step in changing total into serially total.

Let $L(E)$ be a Banach space of measurable functions on a measurable set $E \subset[a, b]$ with natural linear operations. As usual, identify functions equal almost everywhere. Postulate the following on $L(E)$ :

(1) $L(E)$ is contained in $L^{1}(E)$;

(2) $L(E)$ contains the function 1 ;

(3) if $f \in L(E)$, and if for a measurable function $g, 0 \leqq g(x) \leqq f(x)$ almost everywhere, then $g \in L(E)$;

(4) if $f \in L(E)$ and $\chi_{A}$ is the characteristic function of the measurable set $A$, then $\left\|f \chi_{A}\right\| \equiv\|f\|_{\text {.1 }}$ goes to zero as $|A|$ goes to zero, where $|A|$ denotes the Lebesgue measure of $A$.

Example of spaces that satisfy (1)-(4) are the $L^{p}$ spaces $1 \leqq p<\infty$ and the separable Orlicz spaces.

Received by the editors March 30, 1972.

AMS (MOS) subject classifications (1970). Primary 46B15, 46E30; Secondary 41 A30.

Key words and phrases. Schauder basis, completion, series representation.

(c) American Mathematical Society 1974 
THEOREM. Let $\left\{\phi_{k}\right\}_{k=1}^{\infty}$ be a normalized basis for $L(E)$, then given any natural number $N_{0}$ and $\varepsilon>0$, there exists a set $D=D\left(N_{0}, \varepsilon\right)$, contained in $E$ and satisfying $|D|>|E|-\varepsilon$, such that $\left\{\phi_{k}\right\}_{k=N_{0}}^{\infty}$ is serially total in $L(D)$.

We should note that by [1] $\left\{\phi_{k}\right\}_{k=N_{0}}^{\infty}$ is serially total in measure on $E$. We utilize a lemma from [1] as the main tool in the proof of the theorem.

Lemma. Let $\left\{\phi_{k}\right\}_{k=1}^{\infty}$ be a normalized Schauder basis for $L(E), g$ a measurable function finite almost everywhere on $E$. Then given $\varepsilon>0$ and a natural number $N$, there exists a set $e_{0}$ and real numbers $b_{N+1}, \cdots, b_{m}$ such that

$$
\begin{gathered}
e_{0} \subset E, \text { and }\left|e_{0}\right|<\varepsilon ; \\
\left|b_{k}\right|<\varepsilon \text { for } N+1 \leqq k \leqq m ; \\
\left\|\sum_{k=N+1}^{m} b_{k} \phi_{k}-g\right\|_{\left(E \mid e_{0}\right)}<\varepsilon ; \\
\left\|\sum_{k=N+1}^{s} b_{k} \phi_{k}\right\|_{e} \leqq \varepsilon+\|g\|_{e} \text { for all } N+1 \leqq s \leqq m,
\end{gathered}
$$

and every measurable subset e of $E \backslash e_{0}$.

Proof of Theorem. The required set $D$ will be a certain infinite intersection. The individual factors of this intersection are inductively determined.

Choose a sequence of positive terms $\varepsilon_{n}$ with the property

$$
\sum_{n=1}^{\infty} \varepsilon_{n}=\varepsilon
$$

By virtue of the lemma we may choose a set $D_{1}$ whose complement $E_{1}$ has measure less than $\varepsilon_{1}$, and a $\Phi$-polynomial

$$
P_{11}=\sum_{j=v(1,0)+1}^{v(1,1)} b_{j} \phi_{j} \quad \text { where } v(1,0)=N_{0}
$$

satisfying the following conditions:

$$
\begin{aligned}
\left|b_{j}\right|<\varepsilon_{1}, \quad \text { for } v(1,0) & <j \leqq v(1,1), \\
\left\|\phi_{1}-P_{11}\right\|_{D_{1}} & <\varepsilon_{1}, \\
\sup _{s \leqq v(1,1)}\left\|\sum_{j=v(1,0)+1}^{s} b_{j} \phi_{j}\right\|_{e} & <\varepsilon_{1}+\left\|\phi_{1}\right\|_{e}
\end{aligned}
$$

for all measurable subsets $e$ contained in $D_{1}$.

Again applying the lemma twice in succession allows us to choose for 
$i=1,2$, sets $D_{2 i}$ with respective complements $E_{2 i}$ and $\Phi$-polynomials

$$
P_{2 i}=\sum_{j=v(2, i-1)+1}^{v(2, i)} b_{j} \phi_{j}
$$

with $v(1,1)<v(2,0)<v(2,1)<v(2,2)$ such that

$$
\begin{gathered}
\left|E_{2 i}\right|<\varepsilon_{2} / 2 \text { for } i=1,2 ; \\
\left|b_{j}\right|<\varepsilon_{2} \text { if } v(2,0)<j \leqq v(2,2) \\
\left\|\left(\phi_{1}-P_{11}\right)-P_{21}\right\|_{D_{21}}<\varepsilon_{2} / 2 \\
\left\|\phi_{2}-P_{22}\right\|_{D_{22}}<\varepsilon_{2} / 2 \\
\sup _{s \leqq v(2,1)}\left\|\sum_{j=v(2,0)+1}^{s} b_{j} \phi_{j}\right\|_{e}<\varepsilon_{2}+\left\|\phi_{1}-P_{11}\right\|_{e}
\end{gathered}
$$

for all measurable subsets $e$ of $D_{21}$;

$$
\sup _{s \leqq v(2,2)}\left\|\sum_{j=v(2,1)+1}^{s} b_{j} \phi_{j}\right\|_{e}<\varepsilon_{2}+\left\|\phi_{2}\right\|_{e}
$$

for all measurable subsets $e$ of $D_{22}$.

Let $D_{2}^{*}=\bigcap_{i=1}^{2} D_{2 i}$ and $D_{2}=D_{1} \cap D_{2}^{*}$, then $\left|D_{2}\right|>|E|-\sum_{i=1}^{2} \varepsilon_{1}$. By virtue of (12), (13), (6) and the definition of the set $D_{2}$ we obtain

$$
\sup _{s \leqq v(2, k)}\left\|\sum_{j=v(2, k-1)+1}^{s} b_{j} \phi_{j}\right\|_{e} \leqq \begin{cases}\varepsilon_{2}+\varepsilon_{1} & \text { if } k=1 \\ \varepsilon_{2}+1 & \text { if } k=2\end{cases}
$$

for all measurable subsets $e$ of $D_{2}$.

In the $n$th step we apply the lemma successively to the functions

$$
\begin{gathered}
\Psi_{k}=\phi_{k}-\sum_{j=k}^{n-1} P_{j k} \quad \text { with } k=1,2, \cdots, n-1, \\
\Psi_{n}=\phi_{n} .
\end{gathered}
$$

The lemma makes it possible to choose for $k=1,2, \cdots, n$, sets $D_{n k}$ with respective complements $E_{n k}$ and $\Phi$-polynomials

$$
P_{n k}=\sum_{j=v(n, k-1)+1}^{v(n, k)} b_{j} \phi_{j}
$$

where $v(n-1, n-1)<v(n, 0)<v(n, 1)<\cdots<v(n, n)$ such that the following holds:

$$
\begin{gathered}
\left|E_{n k}\right|<\varepsilon_{n} / n ; \\
\left|b_{j}\right|<\varepsilon_{n}, \quad \text { for } v(n, 0)<j \leqq v(n, n) ; \\
\left\|\Psi_{k}-P_{n k}\right\|_{D_{n k}}<\varepsilon_{n} / n ; \\
\sup _{s \leqq v(n, k)}\left\|\sum_{j=v(n, k-1)+1}^{s} b_{j} \phi_{j}\right\|_{e} \leqq \varepsilon_{n}+\left\|\Psi_{k}\right\|_{e}
\end{gathered}
$$


for all measurable subsets $e$ of $D_{n k}$. Let $D_{n}^{*}=\bigcap_{k=1}^{n} D_{n k}$ and $D_{n}=D_{n-1} \cap$ $D_{n}^{*}$, then $\left|D_{n}\right|>|E|-\sum_{k=1}^{n} \varepsilon_{k}$. In analogous fashion to (14) of the second step we obtain in the $n$th step

$$
\sup _{s \leqq v(n, k)}\left\|\sum_{j=v(n, k-1)+1}^{s} b_{j} \phi_{j}\right\|_{e} \leqq \begin{cases}\varepsilon_{n}+\varepsilon_{n-1} & \text { if } k<n ; \\ \varepsilon_{n}+1 & \text { if } k=n ;\end{cases}
$$

for all measurable subsets $e$ of $D_{n}$. Continuing by inductive construction it is easy to see that (18)-(22) holds for each natural number $n$. Define $D=\bigcap_{n=1}^{\infty} D_{n}$, then $|D| \geqq|E|-\sum_{k=1}^{\infty} \varepsilon_{k} \geqq|E|-\varepsilon$.

Now we are ready to show that given any function $f$ in $L(D)$ we can find a series from $\left\{\phi_{j}: v(m, 0) \leqq j \leqq v(m, m), m=1,2, \cdots\right\}$ which will converge to $f$ in the $L(D)$ norm. In fact, if $\sum_{k=1}^{\infty} a_{k} \phi_{k}$ is the Schauder basis expansion of $f$ then $\sum_{j=1}^{\infty} \sum_{k=1}^{j} a_{k} P_{j k}$ converges to $f$ in the $L(D)$ norm.

Let $\delta>0$ be given. Choose $N_{1}$ so that

$$
\left\|\sum_{k=1}^{n} a_{k} \phi_{k}-f\right\|_{D} \leqq\left\|\sum_{k=1}^{n} a_{k} \phi_{k}-f \chi_{D}\right\|<\frac{\delta}{3} \text { for all } n>N_{1} .
$$

Setting $a=\sup _{k}\left|a_{k}\right|$, choose $N_{2}>N_{1}$ so that $a \cdot \varepsilon_{n}<\delta / 3$ for all $n>N_{2}$. By virtue of (20), (15) and the definition of $D$ we obtain

$$
\begin{aligned}
\left\|\sum_{j=1}^{n} \sum_{k=1}^{j} a_{k} P_{j k}-\sum_{k=1}^{n} a_{k} \phi_{k}\right\|_{D} & =\left\|\sum_{k=1}^{n} a_{k}\left(\sum_{j=k}^{n} P_{j k}-\phi_{k}\right)\right\|_{D} \\
& \leqq \sum_{k=1}^{n}\left\|\sum_{j=k}^{n} P_{j k}-\phi_{k}\right\|_{D} \\
& \leqq n \cdot a \cdot \varepsilon_{n} / n<\delta / 3 .
\end{aligned}
$$

Last, choose $N_{3}>N_{2}$ so that

$$
\left|2 \cdot a_{n}\right|<\delta / 3 \text { whenever } n>N_{3} \text {. }
$$

By virtue of (23) and (24) we obtain

Obviously

$$
\left\|\sum_{j=1}^{n} \sum_{k=1}^{j} a_{k} P_{j k}-f\right\|_{D}<\frac{2 \delta}{3}, \text { for all } n>N_{3}
$$

$$
\left\|\sum_{j=1}^{n} \sum_{k=1}^{j} a_{k} P_{j k}+\sum_{k=1}^{n+1} a_{k} P_{n+1 k}-f\right\|_{D}<\frac{2 \delta}{3} \text { for ail } n>N_{3} .
$$

If we add in only part of the second sum; that is,

$$
\sum_{k=1}^{m} a_{k} P_{n+1 k} \quad \text { with } m<n+1
$$


then it is easy to see from (20) that the basis elements $\phi_{i}, i=1,2, \cdots, m$, will be approximated better than before, by $\varepsilon_{n+1} /(n+1)$ instead of by $\varepsilon_{n} / n$. Hence via the calculations in (24), and by (26) it is immediate that

$$
\left\|\sum_{j=1}^{n} \sum_{k=1}^{j} a_{k} P_{j k}+\sum_{k=1}^{m} a_{k} P_{n+1 k}-f\right\|_{D}<\frac{2 \delta}{3} .
$$

Finally, if we add to the summations in (28) only part of the $\Phi$-polynomial $a_{m+1} P_{n+1} m+1$, let us say

$$
\sum_{j=v(n+1, m)+1}^{s} a_{m+1} b_{j} \phi_{j} \text { where } v(n+1, m)<s<v(n+1, m+1)
$$

then (22) and (25) in addition to (28) give us

$$
\left\|\sum_{j=1}^{n} \sum_{k=1}^{j} a_{k} P_{j k}+\sum_{k=1}^{m} a_{k} P_{n+1 k}+\sum_{j=v(n+1, m)+1}^{s} a_{n+1} b_{j} \phi_{j}-f\right\|_{D}<\delta .
$$

Thus, we obtain the desired series convergence. Furthermore, the coefficients of $\phi_{j}$ go to zero, since the $a_{n}$ are bounded by $a$ and the $b_{j}$ go to zero.

\section{BIBLIOGRAPHY}

1. Ben-Ami Braun, An extension of a result by Talalyan on the representation of measurable functions by Schauder bases, Proc. Amer. Math. Soc. 34 (1972), 440-446. MR 45 \#4138.

2. A. A. Talaljan, On the convergence almost everywhere of subsequences of partial sums of general orthogonal series, Izv. Akad. Nauk Armyan SSR Fiz.-Mat. Nauk 10 (1957), no. 3, 17-34. (Russian) MR 19, 742.

3. - The representations of measurable functions by series, Uspehi Mat. Nauk 15 (1960), no. 5 (95), 77-141 = Russian Math. Surveys 15 (1960), no. 5, 75-136. MR 23 \#A2704.

Department of Mathematics, University of South Florida, Tampa, Florida 33620 